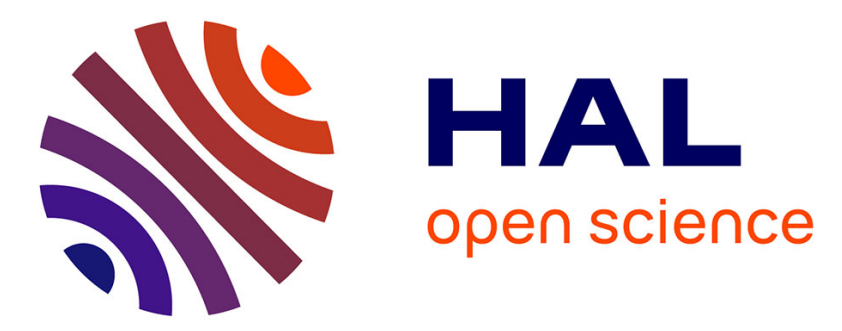

\title{
Hysteresis and instabilities in a spheroid in precession near the resonance with the tilt-over mode
}

\author{
C Nobili, Patrice Meunier, Benjamin Favier, Michael Le Bars
}

\section{To cite this version:}

C Nobili, Patrice Meunier, Benjamin Favier, Michael Le Bars. Hysteresis and instabilities in a spheroid in precession near the resonance with the tilt-over mode. J.Fluid Mech., inPress, 10.1017/jfm.2020.938 . hal-03009224

\section{HAL Id: hal-03009224 \\ https://hal.science/hal-03009224}

Submitted on 17 Nov 2020

HAL is a multi-disciplinary open access archive for the deposit and dissemination of scientific research documents, whether they are published or not. The documents may come from teaching and research institutions in France or abroad, or from public or private research centers.
L'archive ouverte pluridisciplinaire HAL, est destinée au dépôt et à la diffusion de documents scientifiques de niveau recherche, publiés ou non, émanant des établissements d'enseignement et de recherche français ou étrangers, des laboratoires publics ou privés. 


\title{
Hysteresis and instabilities in a spheroid in precession near the resonance with the tilt-over mode
}

\author{
C. NOBILI $I^{1}$, P. MEUNIER ${ }^{1}$, B. F A VIER $\mathbf{R}^{1}$ \\ and M. L E B A R S ${ }^{1}$ \\ ${ }^{1}$ CNRS, Aix-Marseille Univ, Centrale Marseille, IRPHE UMR 7342, 49 rue Joliot-Curie, \\ 13013 Marseille, France
}

(Received $\mathrm{xx}$; revised $\mathrm{xx}$; accepted $\mathrm{xx}$ )

This study explores experimentally the flows driven by precession in an oblate spheroid, in the vicinity of the possible resonance with the tilt-over mode. Two main phenomena are reported, combining observations and velocity measurements. First, an hysteretic cycle is quantitatively described between two uniform vorticity solutions, in good agreement with the historical analytical study of Busse (1968). We then address the destabilization of each branch at low enough Ekman number. We confirm the possible presence of a so-called Conical Shear Instability, recently depicted in the sphere by Lin et al. (2015) and in the spheroid by Horimoto et al. (2020). However, available measurements in the accessible parameter range are not sufficient to definitively discard an elliptical or shear origin of the excited instabilities in the spheroid, as first introduced by Kerswell (1993).

\section{Introduction}

Precession corresponds to the continous rotation of the direction of the spin axis of any rotating body, like e.g. a spinning top. It has been largely studied in fluid mechanics, both because of the large range of possible applications in industrial and natural flows (see e.g. Vanyo 1993), but also because of its appeal from a fundamental point of view: there is indeed a striking contrast between the apparent simplicity of the precession forcing and the complexity of the excited flows. Even at weak forcing amplitude, various types of boundary and bulk instabilities, as well as waves, zonal flows and turbulence, are excited in all investigated geometries, including the cylinder (e.g. Gans 1970; Meunier et al. 2008; Herault et al. 2015), the cube (e.g. Goepfert \& Tilgner 2016), the sphere (e.g. Kida 2011; Boisson et al. 2012; Goto et al. 2014; Lin et al. 2015), the spherical shell (e.g. Triana et al. 2012; Cébron et al. 2019), the spheroid (e.g. Kerswell 1993; Kida 2020; Horimoto et al. 2020) and the ellipsoid (e.g. Cébron et al. 2010; Noir \& Cébron 2013). Numerous open questions remain regarding the flow forced by precession, and in particular we still lack experimental confirmation of the various theoretically suggested processes for instability (see e.g. Le Bars et al. 2015).

Here we focus on precession in an oblate spheroid, which has been studied for more than one century (Sloudsky 1895; Hough 1895) because of its relevance for planetary dynamics, in particular for the Earth's core dynamics and, possibly, its magnetic field generation (e.g. Bullard 1949; Malkus 1968; Vanyo et al. 1995; Noir et al. 2001; Lorenzani \& Tilgner 2001). The analytical solution first given by Sloudsky (1895) and then by Poincaré (1910) for an inviscid flow, is characterized by a uniform vorticity. The precession, spin and fluid axes are in the same plane, and the inclination of the fluid rotation axis depends on the geometry and on the Poincaré number, defined as the ratio of the precession 
to spin rate. This so-called Poincaré's solution is however not unique due to the lack of boundary conditions. The classical hypothesis then consists in considering that the component of the fluid rotation along the spin axis has the same amplitude as the container rotation. The solution diverges when the Poincar number reaches a specific, negative value related to the container oblateness and precession angle, a mechanism explained by the resonance of Poincaré's solution with the so-called tilt-over inertial mode of the rotating fluid (Greenspan 1968). Accounting for the viscosity is necessary for an accurate, regularized solution: this was first done by Busse (1968), who extended Poincaré's work by considering the interaction of the interior solid-body rotation with the Ekman boundary layer, following Stewartson \& Roberts (1963). The three components of the fluid solid-body rotation are then solutions of a system of three non-linear, coupled equations, which was also recovered by Noir et al. (2003) using a less mathematical approach.

Similarly to Poincaré's solution, Busse's solution exhibits a specific behavior in the vicinity of its resonance with the tilt-over eigenmode of the rotating fluid. Depending on the value of the Ekman number (i.e. the ratio of viscous to Coriolis forces), three separate solutions for the fluid vorticity vector might be possible at a given resonant Poincaré number: (i) a weakly inclined state which is the relevant solution at small precession forcing, where the fluid rotation axis is close to the container axis and close to the plane comprising the container and the precession axes; (ii) a strongly inclined state with three vorticity components of similar amplitude, which is relevant for more negative Poincaré numbers; and (iii) an unstable solution inbetween. Bistability and hysteresis between the two former states are thus expected, as latter studied theoretically in full details by Cébron (2015). Hysteresis was indeed observed using torque measurements in the seminal experiment by Malkus (1968), for a precession angle of $96^{\circ}$ in his notations, corresponding here to a precession angle of $84^{\circ}$ with a retrograde precession. Actually, in the limit of a large precession angle (i.e. close to $90^{\circ}$ ), Busse (1968) solution also predicts a second resonance, this time for prograde precession (i.e. a positive Poincaré number): associated bistability and hysteresis were observed in the recent experiment by Horimoto et al. (2018), for a precession angle of $90^{\circ}$. But to the best of our knowledge, hysteresis around the retrograde resonance at moderate precession angle has not been experimentally observed yet, despite careful exploration using ultrasonic Doppler anemometry by Noir et al. (2003), who otherwise closely recovered Busse (1968) predictions.

Beyond the constant vorticity base flow and its dynamics, the route to turbulence for precessing flows in spheroids is still an open question, of fundamental importance for planetary applications (Kerswell 1996). Many instabilities are possible to trigger turbulence. First, the Ekman boundary layer which reconnects the inclined rotation of the fluid with the imposed rotation of the container is subject to a strong shear: it might thus become unstable, as described numerically by Lorenzani \& Tilgner (2001, 2003). Then, Busse (1968) also showed in his theoretical study that the Ekman layer erupts at critical latitudes and generates in the bulk a vertical, geostrophic, shear layer: this localized and intense jet could also destabilize following a classical shear instability, as suggested in the experimental studies of Malkus (1968) and Vanyo et al. (1995). Ekman layer eruption also generates oscillating conical shear layer in the bulk, with an azimuthal wavenumber $m=1$ : this periodic forcing can excite a parametric resonance with two inertial waves of the rotating fluid, as carefully demonstrated in the sphere by Lin et al. (2015) and in good agreement with the experimental results of Goto et al. (2011) (but see also Kida (2019)). This mechanism, called the Conical Shear Instability, has recently been confirmed experimentally in a precessing spheroid by Horimoto et al. (2020). Finally, matching the inclined Busse's constant vorticity solution with the spheroidal boundary 
(a)
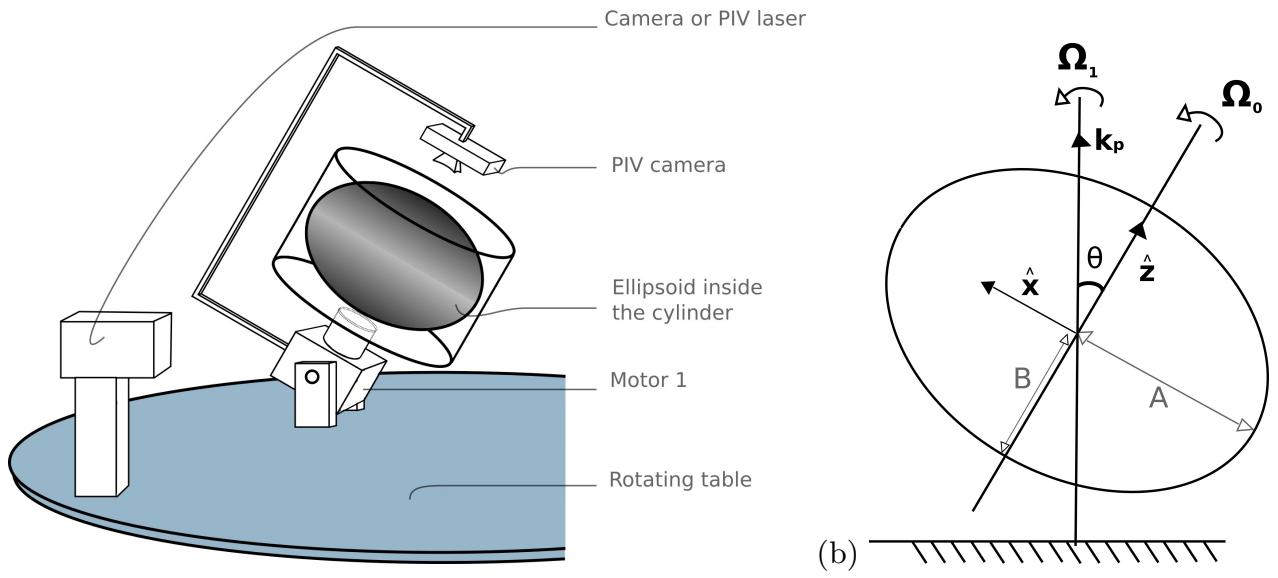

Figure 1. Sketch of the experimental setup. A spheroid of equatorial radius $A$ and minor axis $B$ is filled with water and put inside a cylinder which rotates at the angular velocity $\Omega_{0}$ around its axis $\hat{z}$. The spheroid is laying on a turntable and is tilted with an angle $\theta$ with respect to the axis $\boldsymbol{k}_{p}$ of the platform. The turntable rotates at an angular velocity $\Omega_{1}$ along $\boldsymbol{k}_{p}$ which is vertical here. A laser and cameras also lay on the turntable for PIV measurement or mica-particles observations.

of the container induces two corrections to the otherwise circular streamlines (Kerswell 1993): an elliptical distortion with azimuthal wavenumber $m=2$, and a shear due to their misaligned centers with azimuthal wavenumber $m=1$. As described analytically by Kerswell (1993) and later extended by Wu \& Roberts (2011), both corrections are also capable of sustaining a parametric instability, which has nevertheless never been convincingly observed in a spheroid, except in numerical simulations with adhoc boundary conditions to prevent other types of instability (Lorenzani \& Tilgner 2003; Wu \& Roberts 2009).

The goal of this paper is to provide a better understanding of the base flow and instabilities excited inside an oblate spheroid in precession near the resonance with the tilt-over mode, using laboratory experiments. Material and methods are described in section 2. Section 3 then provides an experimental validation of the hysteretic cycle between two Busse (1968) solutions, as theoretically described by Cébron (2015). In section 4, we investigate the bulk destabilization of those two solutions, trying to decipher between the various theoretical models for the origin of the involved instability (Kerswell 1993; Lin et al. 2015). Section 5 finally gives a short overview of our results as well as some possible targets for future experimental investigations.

\section{Material and methods}

\subsection{Experimental setup}

In this paper, we study the flow of an incompressible fluid inside an ellipsoid of revolution (or spheroid) in precession. The experimental setup is described in figure 1 . A spheroid of equatorial semi-major axis $A=8.5 \mathrm{~cm}$ and axial semi-minor axis $B=7.2$ $\mathrm{cm}$ rotates at the angular velocity $\Omega_{0}$ around its axis and is mounted on a platform which rotates at the angular velocity $\Omega_{1}$. The oblateness of the spheroid $\eta$ defined by $\eta=1-B / A$ is equal to 0.15 . The oblateness is constant in our whole study. The spheroid is filled with water with a kinematic viscosity $\nu$ known within $2 \%$ from its temperature measurement. The spheroid is carved inside a cylinder in order to minimize the optical deformations when viewing along the cylinder's axis. The angular velocities 
of the spheroid and of the rotating platform can be varied independently. The angular velocity $\Omega_{0}$ can vary between 0.25 and $48 \mathrm{rad} . \mathrm{s}^{-1}$ and is measured with an accuracy of $0.1 \%$. The angular velocity of the platform $\Omega_{1}$ can be varied from 0.01 to 6 rad.s ${ }^{-1}$ with increments of 0.01 rad.s ${ }^{-1}$ and is measured with an accuracy of $0.2 \%$. The cylinder axis is tilted relative to the axis of the platform with an angle $\theta$, which can be increased up to $30^{\circ}$ and which is determined with an absolute accuracy of $\pm 0.5^{\circ}$. The inclination angle $\theta$ can be modified while the platform is rotating thanks to a small motor mounted on the platform and controlled externally. It should be noted that the spheroid is off-centered with respect to the axis of the platform, which does not influence the flow in the absence of free surface and density variations. Indeed, it simply adds a centrifugal force which is automatically compensated by a pressure gradient.

Two types of measurements are performed. The first one is qualitative and consists of seeding the flow with flat reflective particles, which are known to preferentially align with the local shear. Mica particles of average diameter of $50 \mu \mathrm{m}$ covered with a titanium dioxide layer to improve reflections are used. A laser diode is used to illuminate a meridian plane of the spheroid and a camera is used to record images at 25 frames per second. The second type of measurement is Particle Image Velocimetry (PIV), which is performed by seeding the flow with passive particles of diameter $40 \mu \mathrm{m}$ and density 0.996 . The particles are illuminated with a thin light sheet created by two $170 \mathrm{~mJ}$ yttrium aluminum garner pulsed lasers. A cylindrical lens is used to provide this laser sheet. Two small mirrors are also added to redirect the rays in the shadows of the luminous cross-section (due to the refraction of the rays on the cylinder). The laser sheet is fixed relative to the rotating table frame (i.e. the precessing frame) and perpendicular to the axis of the spheroid. The height $H$ between the laser sheet and the equatorial plane of the spheroid can be adjusted. The experiments were performed at several fixed heights measured from the equatorial plane between $H=0.2 \mathrm{~cm}$ and $H=3 \mathrm{~cm}$. A video camera mounted on the rotating platform and aligned with the axis of the spheroid is used to record the PIV images. To obtain the velocity field, two successive images are first rotated around the centre of the spheroid to remove the mean solid body rotation of the flow: this increases the accuracy of the measurements. The images are then treated by a cross-correlation algorithm detailed in Meunier \& Leweke (2003). The solid body rotation is finally added, leading to the transverse velocity field and the axial vorticity. It should be noted that the mean solid body rotation of the flow is in general smaller than the angular velocity $\Omega_{0}$ of the spheroid. It is unknown before the PIV measurement and it was thus adjusted by trials and errors for each experiment. There is a radial cut-off on the images at $0.95 R$ (where $R$ is the radius of the boundary on the images) beyond which the images are reconstructued by mirror symetry using $I(0.95 R+\varepsilon)=I(0.95 R-\varepsilon)$. This prevents strong spurious vectors due to the presence of the boundary. The camera and the pulsed lasers are set up so the time $d t$ between the two pulses can be adjusted between 1 and $60 \mu s$. The value of $d t=0.08 / \Omega_{0}$ was the optimal choice since it allowed for a maximal displacement by the flow perturbations, while preventing a too large displacement by the solid body rotation. The experiments were performed at two different frequencies of acquisition: $24 \mathrm{~Hz}$ (i.e. maximal frequency of the camera) and $23 \mathrm{~Hz}$ to quantify any stroboscopic effect for the fast modes. For example, let us assume that a peak is measured in the $23 \mathrm{~Hz}$ spectrum at the frequency $f_{23}$ and at the frequency $f_{24}$ in the $24 \mathrm{~Hz}$ spectrum. If $f_{23}=f_{24}$, it means that there is no stroboscopic effect. But if the two frequencies $f_{23}$ and $f_{24}$ are different, it means that the true frequency $f$ of the peak verifies $f=f_{23}+23 n=f_{24}+24 n^{\prime}$ with $n$ and $n^{\prime}$ integers. 


\subsection{Theoretical formulation and dimensionless parameters}

The flow inside the spheroid is considered incompressible and the kinematic viscosity $\nu$ of the fluid is assumed constant. The equations of motion are written in the precessing frame, i.e. rotating at $\Omega_{1}$. However, the axes are tilted so that the $\hat{\boldsymbol{z}}$ axis corresponds to the (steady) axis of the spheroid. The Navier-Stokes equations are then:

$$
\begin{gathered}
\frac{\partial \boldsymbol{U}}{\partial t}+\boldsymbol{U} \cdot \boldsymbol{\nabla} \boldsymbol{U}+2 \boldsymbol{\Omega}_{1} \times \boldsymbol{U}=-\nabla \Pi+\nu \nabla^{2} \boldsymbol{U} \\
\boldsymbol{\nabla} \cdot \boldsymbol{U}=0 .
\end{gathered}
$$

where $k_{p}$ corresponds to the steady unit vector aligned with the platform's axis (see Fig. 1b). Both PIV measurements and particles visualisations are made in this frame of reference. The centrifugal force is included in a reduced pressure term $\Pi=p / \rho+\mid \boldsymbol{\Omega}_{1} \times$ $\left.\boldsymbol{r}\right|^{2} / 2$, where $p$ is the pressure, $\rho$ the constant fluid density and $\boldsymbol{r}$ the vector position. The boundary conditions are no-slip so that the velocity at the boundaries of the spheroid matches that of the spheroid. By taking the semi-major axis $A$ as the reference length scale and $\Omega_{0}^{-1}$ as the reference time scale, the non-dimensionalized equations are:

$$
\begin{gathered}
\frac{\partial \boldsymbol{u}}{\partial t}+\boldsymbol{u} \cdot \nabla \boldsymbol{u}+2 P_{o} \boldsymbol{k}_{p} \times \boldsymbol{u}=-\nabla \Pi+E \nabla^{2} \boldsymbol{u}, \\
\nabla \cdot \boldsymbol{u}=0,
\end{gathered}
$$

where we have introduced the Ekman number $E=\nu /\left(\Omega_{0} A^{2}\right)$ and the Poincaré number $P_{o}=\Omega_{1} / \Omega_{0}$.

Following Busse (1968), the global torque balance for steady solutions is given by

$$
2 P_{o} \int_{V} \boldsymbol{r} \times\left(\boldsymbol{k}_{p} \times \boldsymbol{u}\right) \mathrm{d} V=-\int_{S} \Pi \boldsymbol{r} \times \boldsymbol{n} \mathrm{d} S+E \int_{V} \boldsymbol{r} \times \nabla^{2} \boldsymbol{u} \mathrm{d} V
$$

where $\boldsymbol{r}$ is the vector position, $V$ is the volume, $S$ is the spheroidal surface and $\boldsymbol{n}$ is the surface normal pointing outward. The precessional torque is balanced by the pressure and viscous torques. In the spherical case, the pressure torque vanishes which is not true in our spheroidal case. Looking for uniform vorticity solution to equation (2.3) of the form $\boldsymbol{\Omega}_{F} \times \boldsymbol{r}$, Busse (1968) derived an implicit equation for the fluid rotation vector $\boldsymbol{\Omega}_{F}$ (Busse 1968; Noir et al. 2003):

$$
\boldsymbol{\Omega}_{F}=\frac{X \Omega_{F}^{2} P_{o}}{X^{2}+Y^{2}} \sin \theta \hat{\boldsymbol{x}}+\frac{Y \Omega_{F}^{2} P_{o}}{X^{2}+Y^{2}} \sin \theta \hat{\boldsymbol{y}}+\Omega_{F}^{2} \hat{\boldsymbol{z}},
$$

with

$$
\begin{gathered}
X=0.259\left(\frac{E}{\Omega_{F}}\right)^{1 / 2}+\eta \Omega_{F}^{2}+P_{o} \cos \theta, \\
Y=2.62\left(E \Omega_{F}\right)^{1 / 2},
\end{gathered}
$$

where $\Omega_{F}=\left|\Omega_{F}\right|$. Note that this solution is only valid in the limit of small Ekman numbers and small oblateness $\eta$.

\section{Hysteretic cycle of Busse's solution}

For high enough precession angle and oblateness, and small enough Ekman number, the steady flow given by Busse (1968) splits into three different solutions, which have been studied theoretically by Cébron (2015). The main objective of this section is to address 
(a)
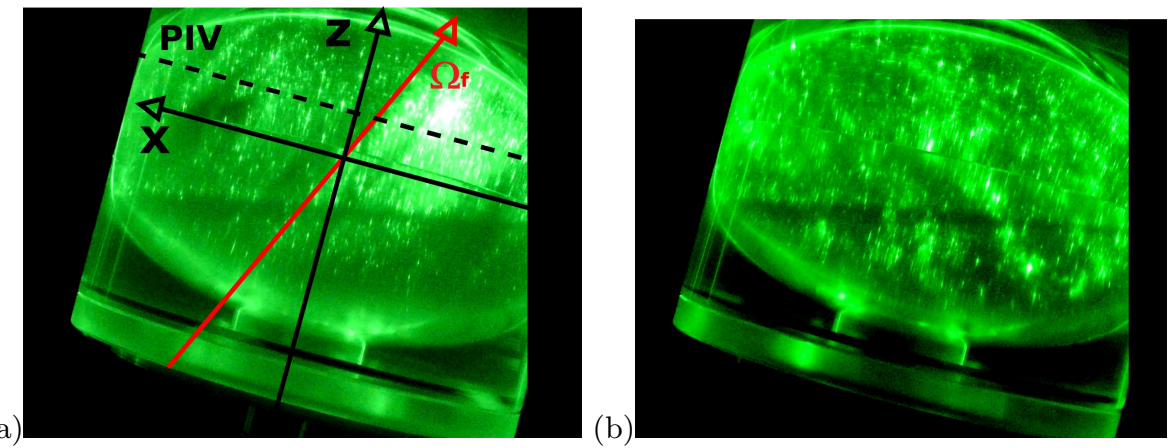

Figure 2. Visualisations of the flow with mica particles in a vertical plane lit with a laser sheet for $E=2.3 \times 10^{-5}$ and $\theta=15^{\circ}$. (a) $P_{o}=-8.7 \times 10^{-2}$ (see also movie VisuS1.avi in supplementary material) and (b) $P_{o}=-9.1 \times 10^{-2}$ (see also movie VisuS1toS2.avi for the transition between the two regimes). The position of the PIV plane along the $z$ axis is shown by the dotted line. The qualitative direction $\boldsymbol{\Omega}_{F}$ around which the fluid rotates is shown by the red arrow.

the stability of those solutions, including the bistability and associated hysteretic cycle theoretically described by Cébron (2015).

The precession angle is fixed at $\theta=15^{\circ}$ while the Poincaré and Ekman numbers are systematically varied. We start by qualitatively observing the mean solid body rotation with the Mica particles method as shown in figure 2 and previously done by Noir et al. (2003). As shown in figure 2 (a) for $E=2.3 \times 10^{-5}$ and $P_{o}=-8.7 \times 10^{-2}$, the axis of rotation of the fluid is tilted towards the direction opposite to the axis $\hat{\boldsymbol{x}}$ (see also movie VisuS1.avi in supplementary material). When $P_{o}$ is shifted to a value of $P_{o}=$ $-9.1 \times 10^{-2}$, as shown in figure 2(b) (and in movie VisuS1toS2.avi), the flow looks qualitatively different and it is possible to discern an axis of rotation tilted towards $+\hat{\boldsymbol{x}}$. Additionally, unsteady small-scale fluctuations are also evidenced by the rapid motion of mica particles, as shown in the movies associated with these two figures. When $P_{o}$ is increased back to its initial value, the axis of rotation of the fluid remains tilted towards the $+\hat{\boldsymbol{x}}$ direction, until we reach the value $P_{o}=-6.1 \times 10^{-2}$ for which the axis of rotation shifts back to an inclination towards $-\hat{\boldsymbol{x}}$ (see movie VisuS2toS1.avi in supplementary material). A transition from two different flows is observed and for this particular angle of precession of $15^{\circ}$, the bistability between the two flows is very clear.

To quantify the bistability better, we now rely on PIV measurements in a narrow region of parameters. As in previous studies (Poincaré 1910; Busse 1968; Noir et al. 2003; Cébron 2015), we assume that the flow essentially consists of a solid body rotation around an axis $\Omega_{F}=\left(\Omega_{x}, \Omega_{y}, \Omega_{z}\right)$, whose components we would like to measure experimentally. The PIV measurements are made in a plane located at a dimensionless distance $h=H / A=0.28$ above the equatorial plane of the spheroid. An example of such measurement is shown in figure 3(a) where one can observe the velocity vector field in the PIV plane. The azimuthal velocity along a diameter (passing through the center of the solid body rotation) is also plotted in figure 3(b). Despite some weak variations, the measured velocity profile is very close to linear confirming the solid body nature of the rotation in the first regime. Figure 3(c) shows the azimuthal velocity in the second regime. It remains very close to a linear profile, with fluctuations smaller than $10 \%$ of the velocity of the spheroid. It indicates that the flow is again very close to a solid body rotation despite the presence of small-scale structures in the flake visualisations of Fig. 2(b) and the associated movie. Note that the center of rotation is not at the center of the cross-section because $\Omega_{F}$ is tilted with respect to the $\hat{\boldsymbol{z}}$ axis and the measurements 

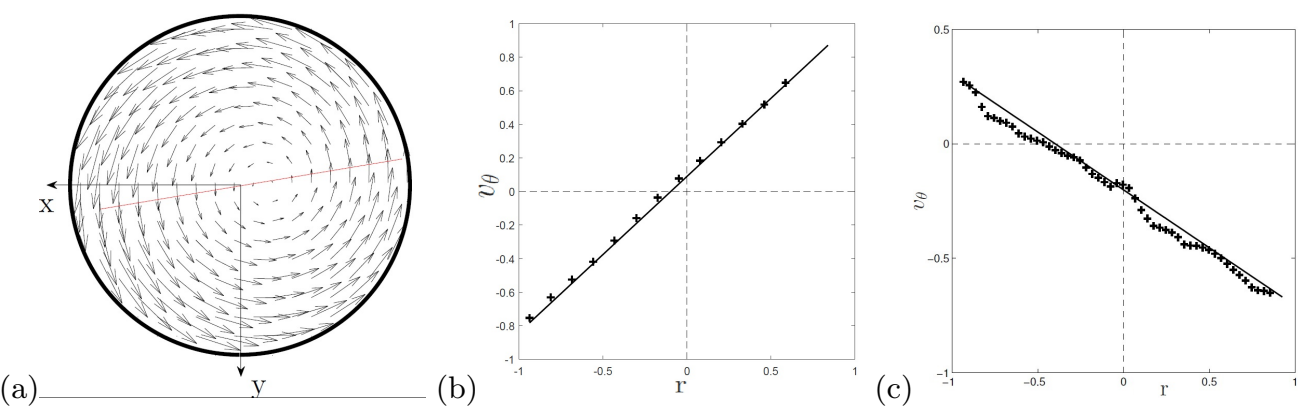

Figure 3. (a) Velocity field obtained by PIV in the plane $z=h=H / A=0.28$ for the first regime, at $E=2.3 \times 10^{-5}, P_{o}=-8.7 \times 10^{-2}$ and $\theta=15^{\circ}$. Only $1 / 4$ of the total number of vectors are plotted for clarity. (b) Symbols show the azimutal velocity along the red segment plotted in (a), with $r$ the coordinate along the red axis oriented towards increasing $x$ and with $r=0$ corresponding to the spheroid axis. (c) Azimuthal velocity profile for the second regime at $E=2.3 \times 10^{-5}, P_{o}=-8.9 \times 10^{-2}$ and $\theta=15^{\circ}$. In (b) and (c), the solid line corresponds to the theoretical velocity profile for a solid body rotation fitted from the experimental velocities.

are made above the equator. It is possible to fit the experimental $2 \mathrm{D}$ velocity field with a three-dimensional solid body rotation $\Omega_{F} \times \boldsymbol{r}$ where $\boldsymbol{r}$ is the position vector, using a standard least-square approach. In the plane of measurement $z=h$, this leads to :

$$
\left[\begin{array}{l}
u_{x} \\
u_{y}
\end{array}\right]=\left[\begin{array}{c}
-y \Omega_{z}+h \Omega_{y} \\
x \Omega_{z}-h \Omega_{x}
\end{array}\right] .
$$

A very good agreement is obtained between the experimental and the fitted azimutal velocities, as shown in figure $3(\mathrm{~b})$ with an error of the order of a few percent.

Now that we can accurately measure the average orientation of the fluid rotation, we systematically vary the Poincaré number for a fixed Ekman number $E=2.3 \times 10^{-5}$. Results are shown in figure 4 . We first discuss the transition as $P_{o}$ is decreased, shown by the circles in figure 4 . The Poincaré number is gradually modified while the experiment is running by adjusting the rate of precession $\Omega_{1}$. We ensure that each solution has reached a steady state by waiting for at least 600 rotation periods between each modification of the Poincaré number. As $P_{o}$ is decreased to a critical value of $P_{o}=-9.1 \times 10^{-2}$, the solid body rotation suddenly shifts to another solution. The first solution (S1 in the following) is the closest to a solid body rotation aligned with the spheroid : $\Omega_{z}$ is between 0.9 and 1 while $\Omega_{x}$ and $\Omega_{y}$ are much smaller. When the second solution (S2 in the following) is reached, all three components of the rotation vector significantly change. When $P_{o}$ is increased back to its initial value (cross symbols in figure 4), the solid body rotation remains on S2 past the critical value $P_{o}=-9.1 \times 10^{-2}$ and finally shifts back to S1 for $P_{o}=-6.3 \times 10^{-2}$. An hysteresis cycle is formed. The transition between S1 and S2 is subcritical.

The observed bistability is correctly described by the viscous Busse's solution, implicitly given by equation (2.4). This equation is solved numerically and results are plotted in figure 4 along with the experimental measurements. Busse's theory plotted in figure 4 in solid lines predicts the hysteretic cycle and is qualitatively consistent with the experimental results. The solution S1 is in red, the solution $\mathrm{S} 2$ in blue, while a third solution, not observed experimentally, is shown in green. The comparison with the experiments shows a good agreement in the amplitude of the solution S1. For the amplitude of $\mathrm{S} 2$, the agreement is not as clear, in particular for $\Omega_{y}$. The critical $P_{o}$ is in 

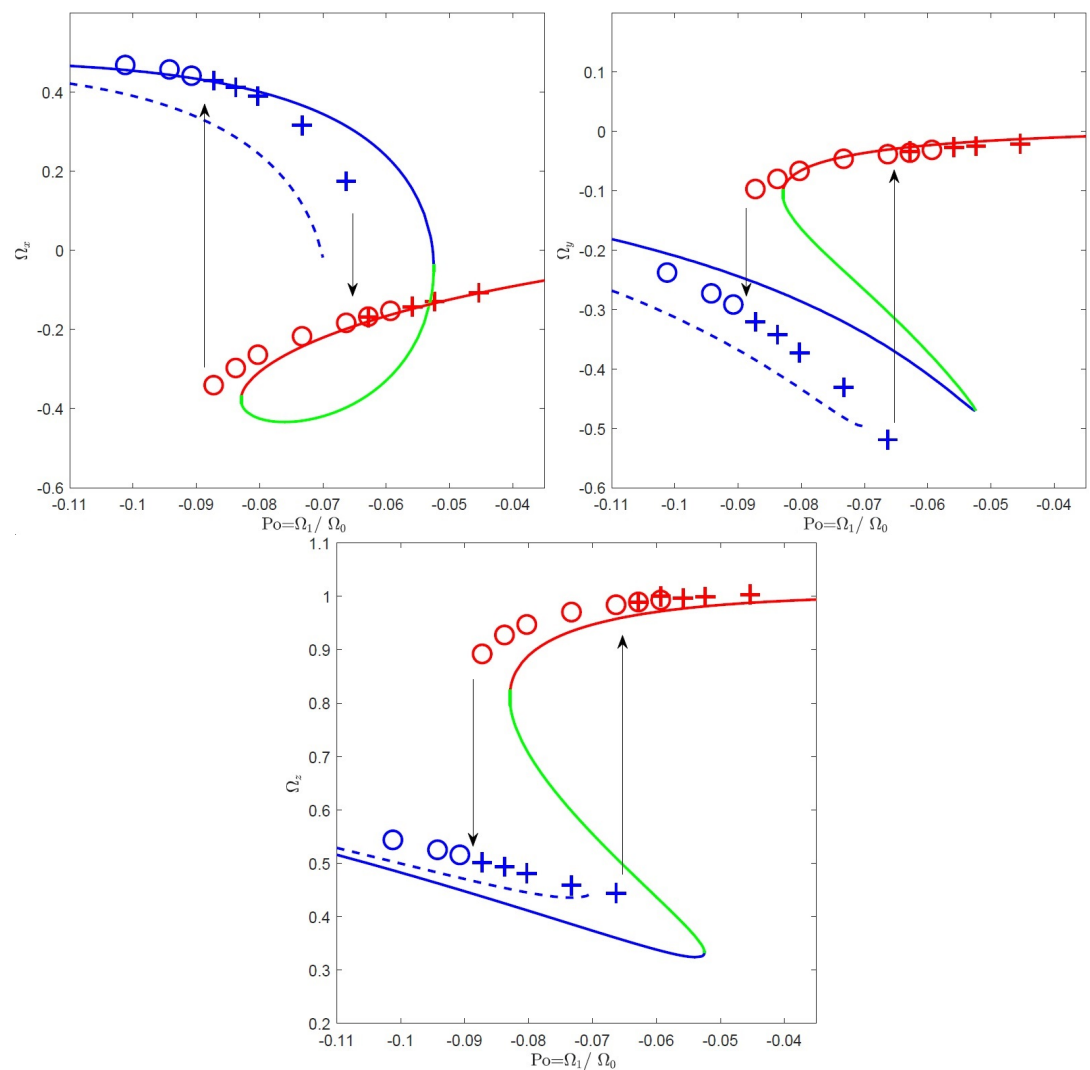

FiguRe 4. Components of the rotation rate $\boldsymbol{\Omega}_{F}$ as a function of the Poincare number for $E=2.3 \times 10^{-5}, \theta=15^{\circ}, h=H / A=0.33$. These experiments correspond to a gradual decrease (symbols $\circ$ ) and to an incremental increase (symbols + ) of the Poincaré number. The continuous lines correspond to the three Busse's solutions obtained numerically. The dotted lines show the Busse's solutions for S2 when accounting for a turbulent boundary layer with a torque given by (3.2) rather than $(2.4 \mathrm{c})$. The arrows indicate the transitions between solutions.

good agreement for the $\mathrm{S} 1 \rightarrow \mathrm{S} 2$ transition but the $\mathrm{S} 2 \rightarrow \mathrm{S} 1$ transition occurs earlier in the experiments.

A first possible explanation for the departure from Busse's theory is that the boundary layer is turbulent for S2 at this Reynolds number $R e=\left|\hat{\boldsymbol{z}}-\Omega_{F}\right| / \sqrt{E} \sim 150$. Indeed, Sous et al. (2013) observed that the boundary layer becomes turbulent at $R e=150$, which leads to a higher torque on the bulk flow. Following Cébron et al. (2019), one can estimate the torque created by a turbulent boundary layer as

$$
Y=\lambda_{t}\left|\hat{\boldsymbol{z}}-\boldsymbol{\Omega}_{F}\right|^{2}=\frac{45 \pi}{32} \frac{1+\eta^{5}}{1-\eta^{5}} u_{0}^{2} \sqrt{1-\frac{C_{2}^{2} u_{0}^{2}}{k^{2}\left|\hat{\boldsymbol{z}}-\Omega_{F}\right|^{2}}}
$$

where $u_{0}$ is solution of

$$
\frac{u_{0}^{2}}{\left|\hat{\boldsymbol{z}}-\boldsymbol{\Omega}_{F}\right|^{2}}=\frac{k^{2}}{\left[\log \left(u_{0}^{2} /(0.22 E)\right)-C_{1}\right]^{2}+C_{2}}
$$

with $k=0.4$ the Kolmogorov constant and $\left(C_{1}, C_{2}\right)=(3.3,3)$ empirical constants given by Sous et al. (2013) for a plane boundary layer. In our case, for $E=2.3 \times 10^{-5},\left|\hat{\boldsymbol{z}}-\boldsymbol{\Omega}_{F}\right|$ is always close to 0.75 for the second Busse's solution such that the torque created by a 
turbulent boundary layer is always close to 0.0162 . Replacing the laminar viscous torque $Y=-2.62 \sqrt{\Omega_{f} E} \sim 0.0109$ by this turbulent torque in Busse's equation (2.4) leads to a new prediction for the solution $\mathrm{S} 2$ which is plotted as a blue dashed line in figure 4 . This turbulent torque actually corresponds to a turbulent viscosity 2.2 times larger than the molecular value. The experimental results are located between the two theories, which suggests that the boundary layer is not completely turbulent at this Reynolds number $R e \sim 150$, which corresponds to the transition from a laminar to a turbulent boundary layer (Sous et al. 2013). In our experiment, the boundary layer has not been analysed such that it is hard to tell whether it is turbulent or laminar.

Other explanations for this discrepancy could be invoked. First, Eq.(3.58) of Zhang et al. (2014) suggests that the numerical coefficient 2.62 for $Y$ in (2.4c) might decrease by $20 \%$ for our spheroid compared to a sphere. Second, Eq.(3.57) of Zhang et al. (2014) suggests that the numerical coefficient 0.259 for $X$ in (2.4b) might be three times smaller for our spheroid compared to a sphere. However, the induced modifications on $X$ and $Y$ are too small to explain the discrepancy between the laminar Busse's solution and the experiment. Third, as shown by Kida (2018), the critical latitude moves when the precession rate increases. For precession rates much larger than the rotation rate, the eruption of the boundary layer takes place in a ring at the equator of the precession axis. This might modify the structure of the instabilities. However, these singularities have always been neglected in the calculation of the torque from the boundary to the bulk flow. This modification may not play an important role for the base flow. Finally, the visualisations of Fig. 2(b) and the corresponding movie indicate that the bulk flow seems to be more complex than a simple solid body rotation, with small-scale unsteady structures. We will show in the next section that these are inertial modes which may generate an axisymmetric flow by nonlinear streaming, as is well known for a precessing cylinder (Lagrange et al. 2011). This can modify the mean solid body rotation and explain the weak discrepancy between Busse's solution and the experimental results.

Finally, we have systematically varied the Ekman number: both Mica particles experiments and PIV measurements are gathered in figure 5. The domain where S1 and S2 can be observed corresponds to the region with black and green symbols. The hysteresis cycle is only observed for Ekman numbers smaller than $2 \times 10^{-4}$. The bistable region increases in size when $E$ decreases, as predicted theoretically. However, it is a bit smaller than the theoretical region predicted by Busse: as already seen before, for all explored values of Ekman number, the transition from S2 to S1 occurs for smaller values of the Poincar number in the experiments, since the solution $\mathrm{S} 2$ is unsteady.

In conclusion, we have measured the base flow driven by precession in a spheroidal cavity, which can be reasonably approximated by a solid body rotation as assumed by Busse (1968) in the explored parameter range. An hysteresis cycle is observed for a certain range of Poincaré numbers. This bistable regime, obtained here for a tilt angle of $15^{\circ}$, is similar to what has been obtained for a larger tilt angle of $90^{\circ}$ by Malkus (1968) and Horimoto et al. (2018). A good agreement with Busse's theory is obtained when the flow is stationary, which is the case for solution S1 over almost all the parameter space explored. However, the second solution S2 is slightly different from the prediction by Busse, mainly because this solution does not appear to be stationary in the range accessible to our set-up due to the presence of instabilities. It is therefore natural to study now the hydrodynamic stability of these two solutions, which is the main objective of the following section. 


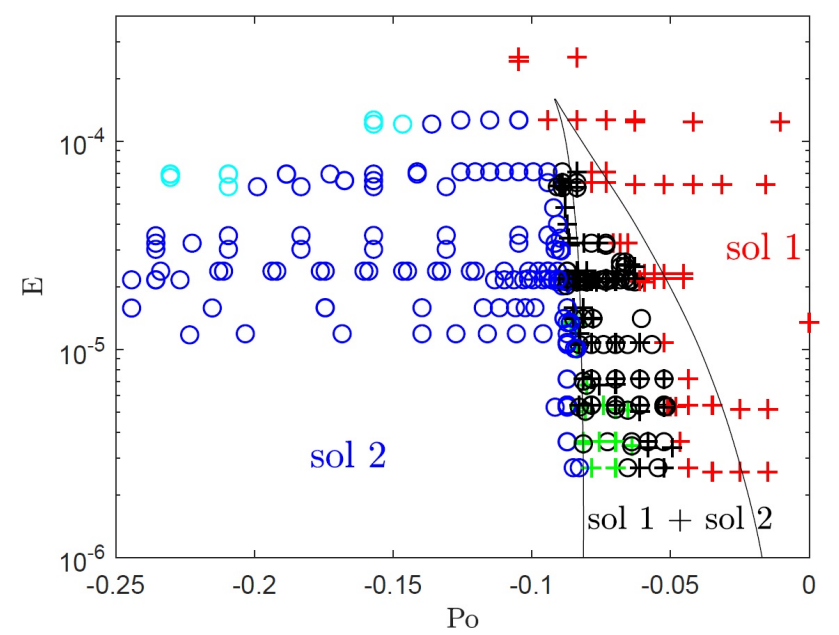

FiguRE 5. Stability diagram of all the experiments as a function of $E$ and $P_{o}$. Crosses correspond to S1 while circles correspond to S2. The hysteresis region corresponds to black and green symbols. This region is compared to Busse's theory solved numerically using the molecular viscosity, and shown by solid lines. For S1, red and black crosses correspond to steady flows, whereas green crosses correspond to unsteady flows. For S2, blue and black circles correspond to unsteady flows, whereas cyan circles correspond to steady flows.

\section{Instabilities of the base flow}

When the Ekman number is sufficiently low, both Busse's solutions can destabilise via hydrodynamic instability leading to a turbulent flow far from the threshold. Two different instabilities have been observed depending on the Poincar and the Ekman number. They are described in the following close to their threshold.

\subsection{Instability of the first Busse's solution}

The first Busse's solution S1 can destabilise when the Ekman number is smaller than about $10^{-5}$ and when the Poincar number decreases beyond about -0.07 . It corresponds to the green crosses in the stability diagram of figure 5. In this regime, the mean flow remains close to the first Busse's solution and does not switch to the second Busse's solution. However, a perturbation arises with a well defined frequency $f_{I}$, as shown in the temporal spectrum of figure 6(a). This spectrum is computed from the spectral density of the vorticity which is averaged over the entire PIV section:

$$
E_{f}=\frac{1}{\pi} \iint_{r<1}|\tilde{\omega}(\boldsymbol{r}, f)|^{2} d \boldsymbol{r}
$$

where the temporal Fourier transform of the vorticity is defined as

$$
\tilde{\omega}(\boldsymbol{r}, f)=\frac{1}{t_{f}-t_{i}} \int_{t_{i}}^{t_{f}} \omega(\boldsymbol{r}, t) \mathrm{e}^{\mathrm{i} 2 \pi f t} d t .
$$

It was found that this quantity reveals the instability better than the spectral density of the velocity. This is probably because Busse's solution contains a uniform vorticity which is insensitive to small displacements and because the instability contains small structures which have a large vorticity even for small velocities.

In figure 6(a), the Fourier transform is computed over the total duration of the PIV measurement, i.e. about 350 rotation periods. It leads to an accurate determination of the frequency of the instability $2 \pi f_{I}=7.99$. However, it is also possible to measure 

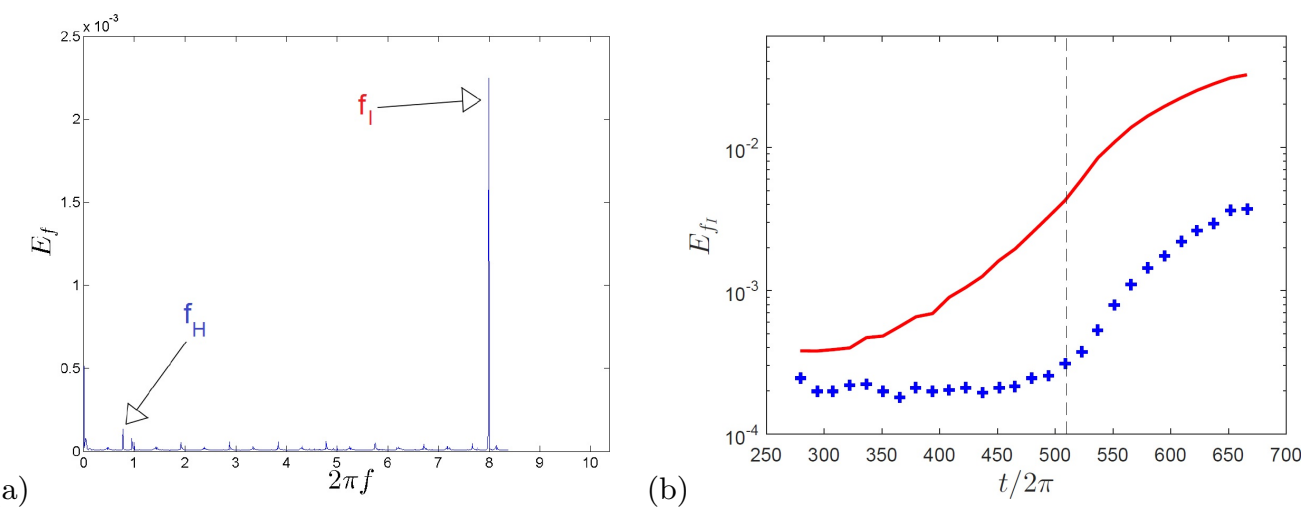

Figure 6. Temporal spectrum (a) and temporal evolution (b) of the spectral density of vorticity defined by (4.1) and measured by PIV at $h=0.065$. The Poincaré number is decreased at $t=0$ from -0.0826 to -0.0838 . In (b), the spectral density is measured at the frequency of the instability $f_{I}$ (in red) and at the frequency of the harmonic $f_{H}$ (in blue). In (b), the duration of the sliding window $t_{f}-t_{i}$ is equal to 14.3 rotation periods in order to be small compared to the growth time of the instability and large compared to the period of the instability (equal to 0.125 rotation period).

the Fourier transform over smaller time intervals in order to plot the spectral density as a function of time. For example, Figure 6(b) shows the temporal variation of $E_{f_{I}}$ at the frequency $f_{I}$ of the instability. In this experiment, the Poincar number has been decreased at $t=0$ by $1.5 \%$ in order to switch from a stable to an unstable regime. The spectral density of vorticity $E_{f_{I}}$ remains constant during about 300 rotation periods, because the signal is smaller than the noise in the measurement. It then grows by two orders of magnitude until 600 rotation periods where it seems to saturate. This is a clear indication that the flow has become unstable when the Poincar number has decreased from -0.0826 to -0.0838 . The growth rate $\sigma$ can be estimated by fitting the spectral density with an exponential function $\mathrm{e}^{2 \sigma t}$ between $t / 2 \pi=430$ and 550 , leading to:

$$
\sigma=1.3 \times 10^{-3} \pm 30 \% \text {. }
$$

It can be noted that the spectrum of figure 6(a) exhibits a small peak at the frequency $2 \pi f_{H}=0.77$. It has been checked experimentally (by doing the same experiment using two different acquisition frequencies $f_{\text {acqu }}$ ) that this frequency is shifted by $2 \pi f_{\text {acqu }}=$ 16.67 because the acquisition frequency of the camera is too low. The real frequency of this peak is in fact equal to $2 \pi f_{H}=-15.9$, which corresponds to the harmonic of the instability. The spectral density at this frequency $f_{H}$ is plotted in figure $6(\mathrm{~b})$ as a function of time. It starts to increase above the noise level after 500 rotation periods and then grows slightly faster than the primary mode, with a growth rate of the order of $\sigma=1.7 \times 10^{-3} \pm 30 \%$.

Figure 7 shows the spatial structure of the vorticity filtered at the frequency $f_{I}$ of the instability. The snapshot is taken towards the end of the exponentially growing regime of the instability in order to get the best signal to noise ratio. The real part of the vorticity $\tilde{\omega}$ exhibits two concentric rings of vortices. The inner ring contains 18 well defined vortices of alternate sign. The second ring, located close to the boundary, is less organized despite its larger amplitude. This is probably due to the interference with the boundaries, which decreases the accuracy of the PIV.

The azimuthal spectrum of the vorticity can be obtained using an azimuthal Fourier 


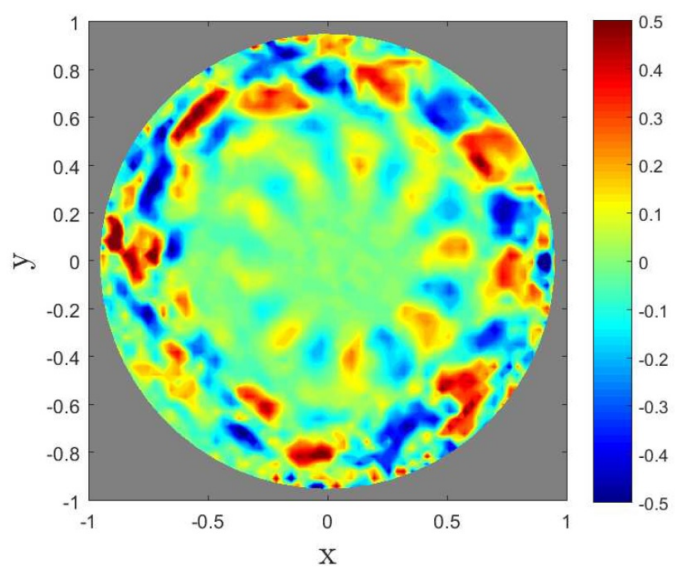

(a)

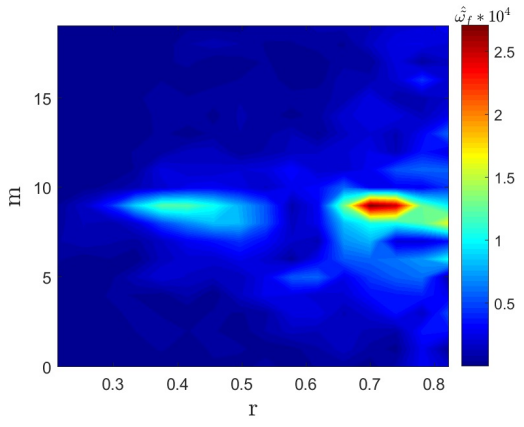

(c)

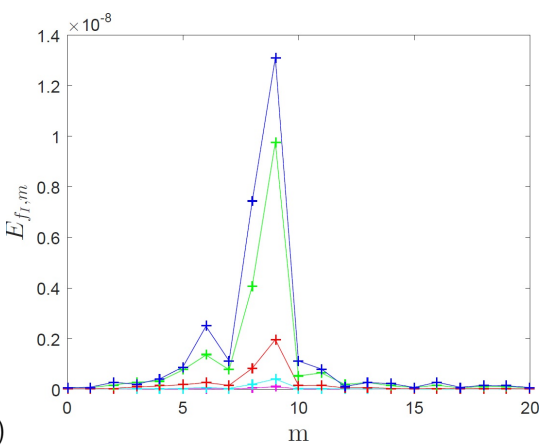

Figure 7. (a) Real part of the vorticity filtered at the frequency of the instability $f_{I}$ as defined by Eq. (4.2). The Fourier transform is calculated over 81.6 rotation periods around $t / 2 \pi=510$ (corresponding to the dashed line of Fig. 6b). (b) Azimuthal spectrum of the vorticity as a function of the radius at $t / 2 \pi=510$. (c) Azimuthal spectrum integrated over $r$, as defined by (4.5) after 306 rotations (purple), after 387 rotations (cyan), after 469 rotations (red), after 551 rotations (green) and after 632 rotations (blue). Lines between the symbols are only here to improve the visibility of the curves. Here, $P o=-0.0838, E=1.3 \times 10^{-5}$ and $h=0.065$.

transform:

$$
\hat{\tilde{\omega}}_{m}\left(r, f_{I}\right)=\int_{\theta=0}^{\theta=2 \pi} \tilde{\omega}\left(r, \theta, f_{I}\right) e^{i m \theta} d \theta .
$$

It is plotted in figure $7(\mathrm{~b})$ for $r$ varying from 0.2 to 0.8 . The two rings of vortices create two lobes around $r=0.4$ and $r=0.7$ with an azimuthal wavenumber close to $m=9$.

By integrating over $r$ this azimuthal spectrum, it is possible to get the total azimuthal spectrum of the vorticity

$$
E_{f_{I}, m}=\int_{r=0}^{r=1}\left|\hat{\tilde{\omega}}_{m}\left(r, f_{I}\right)\right|^{2} r d r
$$

This spectrum is plotted in figure $7(\mathrm{c})$ at different times. All the spectra present a maximum spectral density of vorticity at the azimuthal wavenumber $m=9$. They also exhibit a moderate vorticity (about $50 \%$ of the maximum) at the azimuthal wavenumber $m=8$ and a weak peak (about $20 \%$ of the maximum) at $m=6$. This multi-modal signature clearly points towards a parametric instability involving a given forcing and two inertial modes of our rotating fluid.

Actually, this structure with large azimuthal wavenumbers is very similar to the 
structure which has been observed in a precessing sphere. Lin et al. (2015) argued that it was induced by a parametric resonance between the conical shear layer generated at the critical latitude with an azimuthal wavenumber $m=1$, and two free inertial modes of the sphere with azimuthal wavenumbers $m=17$ and $m=18$. It is thus tempting to relate the instability observed here with $m=9$ and $m=8$ to such a Conical Shear Instability (CSI). However, the presence of a weak peak at $m=6$ indicates that a weak elliptic coupling $m=2$ might also be present, which could transfer some energy from $m=8$ to $m=6$. This coupling could be due to an elliptic instability as described by Kerswell (1993). Furthermore, the presence of the two azimuthal wavenumbers $m=8$ and $m=9$ could also be due to a shear instability described by Kerswell (1993), where two free inertial modes are coupled by the mean shear of the flow with $m=1$. Note that the two latter instabilities cannot be found in a sphere because they rely on the oblateness of the container.

To try to disentangle the various possible origins of the observed instability, it is useful to determine its inviscid growth rate, which we do by measuring the variation of the viscous growth rate with respect to the Poincar number. We thus measure the growth rate accurately (within 20\%) by fitting the growth of the amplitude of modes $m=8$ and $m=9$ (rather than the growth of the total spectral density of vorticity) for two different Poincar numbers. The growth rate increases from $1.5 \times 10^{-3}$ to $7.3 \times 10^{-3}$ when the Poincar number decreases from -0.0838 to -0.0861 , which leads to:

$$
\frac{\partial \sigma_{\text {inv }}}{\partial P o}=-2.5 \pm 30 \% \text {. }
$$

The measured variation corresponds to the variation of the inviscid growth rate because the viscous damping of the instability is independent of the Poincar number: it only depends on the Ekman number.

Experimentally, it is also found that the tilt angle $\theta_{f}$ of the solid body rotation defined by

$$
\tan \theta_{f}=\frac{\sqrt{\Omega_{x}^{2}+\Omega_{y}^{2}}}{\Omega_{z}}
$$

varies as

$$
\frac{\partial \theta_{f}}{\partial P_{O}}=-17 \pm 20 \%
$$

for these Poincar and Ekman numbers. Assuming that the inviscid growth rate is proportional to the tilt angle $\theta_{f}$ (which is a fair assumption since the inviscid gowth rate is proportional to the differential rotation for small tilt angles) gives an empirical formula for the inviscid growth rate $\sigma_{\mathrm{inv}}=0.15 \theta_{f} \pm \mathbf{5 0} \%$. This equation can also be written $\sigma_{\text {inv }}=1.4 E^{1 / 5} \theta_{f} \pm \mathbf{5 0} \%$ if we assume that the inviscid growth rate scales as $E^{1 / 5}$ as in the CSI (Lin et al. 2015). Finally, assuming that the viscous damping effect scales as $\sqrt{E}$ leads to an empirical formula for the total growth rate if it was due to the CSI (by imposing $\sigma=0$ at the threshold where $\theta_{f}=0.32$ ):

$$
\sigma=1.4\left(E^{1 / 5} \theta_{f}-9.3 \sqrt{E}\right) .
$$

For a sphere, it was found experimentally (Goto et al. 2011) and numerically (Lin et al. 2015) that the CSI is unstable if $\left|P_{o} \sin \alpha\right|-25.1 E^{4 / 5}$ is positive. It is thus logical to assume that the growth rate of the CSI is proportional to this term, which can be written as

$$
\sigma_{C S I}=C\left(E^{1 / 2} \theta_{f} / 0.3797-25.1 E^{4 / 5}\right)
$$


since for a sphere, the tilt angle $\theta_{f}$ is given from Busse's solution (see Kida 2013, Eqs. $(4.25,4.26))$ by $\theta_{f} \sqrt{E}=0.3797 P_{o} \sin \alpha$. Multiplying this formula by $0.3797 E^{-3 / 10}$ leads to

$$
\sigma_{C S I}=C^{\prime}\left(E^{1 / 5} \theta_{f}-9.53 \sqrt{E}\right)
$$

with $C^{\prime}=0.3797 C E^{-3 / 10}$. This prediction deduced from the sphere is in excellent agreement with the empirical, experimental formula (4.9) found in this paper for a spheroid. Note that the second term of those equations corresponds to the viscous damping: the value $1.4 \times 9.3 \simeq 13$ is reasonable compared to other viscous damping prefactors determined for similar resonant instabilities, where it ranges from 2.62 for the spin over mode to 20 for more complex modes (Grannan et al. 2014; Lemasquerier et al. 2017). The CSI is thus an excellent candidate to explain the observed instability.

In order to compare the experimental growth rate to the predictions of the elliptic instability and the shear instability, it is necessary to calculate the ellipticity and the shear of the base flow. Following Kerswell (1993), the velocity is written using new coordinates $\left(x^{\prime}, y^{\prime}, z^{\prime}\right)$ where the $z^{\prime}$-axis is aligned with the axis of rotation of the bulk flow:

$$
\boldsymbol{u}=\Omega_{z^{\prime}}\left[\left(-y^{\prime}, x^{\prime}, 0\right)+\beta\left(y^{\prime}, x^{\prime}, 0\right)+\epsilon\left(0, z^{\prime}, 0\right)\right] .
$$

For the normal flow to vanish on the spheroidal surface, the shear must be equal to

$$
\epsilon=\frac{2 \eta_{K} \tan \left(\theta_{f}\right)}{2+\eta_{K}+2 \tan ^{2}\left(\theta_{f}\right)}
$$

and the ellipticity must be equal to

$$
\beta=\frac{2 \eta_{K} \tan ^{2}\left(\theta_{f}\right)}{\left(2+\eta_{K}^{2}\right)+\left(4+2 \eta_{K}\right) \tan ^{2}\left(\theta_{f}\right)},
$$

where $\eta_{K}=\eta(2-\eta) /(1-\eta)^{2}$ is equal to 0.3841 in our experiments.

The variation of the shear can be calculated from (4.13) as $\partial \epsilon / \partial \theta_{f}=-0.27$ at threshold (where $\theta_{f}=0.32$ ). Using (4.6) and (4.8), it leads to the value $\partial \sigma_{\text {inv }} / \partial \epsilon=0.54 \pm \mathbf{5 0} \%$. Finally, assuming that the growth rate is proportional to the shear $\epsilon$ and adding a viscous damping as $\sqrt{E}$ (with a coefficient adjusted such that the growth rate vanishes at $\theta_{f}=$ 0.32 , i.e. at $\epsilon=0.11$ ) gives an empirical formula for the growth rate if it is due to the shear instability:

$$
\sigma=0.54 \epsilon-14.6 \sqrt{E} .
$$

This formula is in fair agreement with the prediction for the shear instability coming from the linear theory of Kerswell (1993), valid in the limit of small oblateness: the first coefficient is expected to be below 0.6 and the second coefficient is expected to vary from 2.6 for the spin-over mode to 20 for complex modes (Grannan et al. 2014; Lemasquerier et al. 2017).

Finally, the variation of the ellipticity can be calculated from (4.14) as $\partial \beta / \partial \theta_{f}=-0.17$ at the threshold (where $\theta_{f}=0.32$ ). Using (4.6) and (4.8), it leads to the value $\partial \sigma_{\text {inv }} / \partial \beta=0.87 \pm \mathbf{5 0} \%$. Finally, assuming that the growth rate is proportional to the ellipticity $\beta$ and adding a viscous damping as $\sqrt{E}$ (with a coefficient adjusted such that the growth rate vanishes at $\theta_{f}=0.32$, i.e. at $\beta=0.032$ ) gives an empirical formula for the growth rate if it is due to the elliptic instability:

$$
\sigma=0.87 \beta-7.7 \sqrt{E} .
$$

This formula is again in fair agreement with the linear prediction for the elliptic instability derived in the limit of small oblateness: the first coefficient is expected to be below 1 , 
depending on the relative rotation of the fluid and of the elliptic distortion (Le Bars et al. 2010), and the second coefficient is expected to vary from 2.6 to 20 (Grannan et al. 2014).

To conclude, it thus seems that the instability observed experimentally on mode S1 can be explained theoretically by the three instabilities: shear, elliptic and conical shear instability. It is actually possible that the three instabilities coexist. It can explain the high azimuthal wavenumbers of the unstable modes, but also the presence of three azimuthal wavenumbers $(\mathrm{m}=9,8$ and 6$)$ separated by $\Delta m=1$ (characteristic of the CSI and the shear instability) and $\Delta m=2$ (characteristic of the elliptic instability).

\subsection{Instability of the second Busse's solution}

The second Busse's solution S2 was found to be unstable in most cases. It corresponds to the blue symbols in the stability diagram of figure 5, which cover a large region of the domain for the solution S2. The only experiments that were found stable for the second Busse's solution correspond to the cyan symbols, which are limited to large Ekman numbers (larger than about $10^{-4}$ ).

At threshold, the instability is characterized by a temporal growth of the spetral density of vorticity at a frequency equal to $2 \pi f_{I}=2.8$. However, the total spectral density of vorticity only increases by a factor 3 between the initial state and the saturated state. This is due to the strong initial noise in the measurement probably related to the strong tilt of the second Busse's solution, and to the early viscous saturation due to the large Ekman number. This does not allow us to measure a growth rate accurately, which will rather be measured on the amplitude of the azimuthal wavenumbers of the instability. It should be noted that a weaker peak of spectral density of vorticity is also measured at $2 \pi f_{H}=5.6$, which corresponds to the harmonic of the instability's frequency.

Figure 8 shows the spatial structure of the vorticity filtered at the frequency $f_{I}$ of the instability at the end of the exponentially growing regime. The real part of the vorticity $\tilde{\omega}$, plotted in figure $8(\mathrm{a})$, exhibits a complex spiraling structure containing about 4 positive and 4 negative vortices. The azimuthal spectrum, defined by (4.4), is plotted in figure 8(b). It indeed confirms the predominance of the azimuthal wavenumber $m=4$ with the presence of two patches extending from $r=0.2$ to 0.4 and from $r=0.5$ to 0.6. Integrating the azimuthal spectrum over $r$ leads to the azimuthal spectrum of total spectral density of vorticity, as plotted in figure $8(\mathrm{c})$. It indicates that the vorticity is significantly larger for azimuthal wavenumbers $m=3$ and $m=4$ than for the other azimuthal wavenumbers. The presence of two azimuthal wavenumbers separated by $\Delta m=1$ is charateristic of the CSI and the shear instability rather than the elliptic instability. However, note that PIV measurements in a plane located above the equator (at $z=0.18$ ) indicate that there is also a peak for $m=6$, wich is about $50 \%$ of the peak for $m=4$.

By fitting the temporal evolution of the amplitude of modes $m=3$ and $m=4$, it is possible to accurately measure the growth rate $\sigma$ of the instability. It was found to be equal to 0.0062 for $P o=-0.157$ and to 0.02 for $P o=-0.147$ with uncertainties of the order of $5 \%$. As before, assuming that the viscous damping is independent of the Poincar number, the variation of the inviscid growth rate can be deduced as:

$$
\frac{\partial \sigma_{\mathrm{inv}}}{\partial P_{O}}=1.4 \pm \mathbf{1 0} \%
$$

As in the previous section, using the fact that $\partial \theta_{f} / \partial P_{O}=3.4 \pm \mathbf{2 0} \%$ at the threshold 


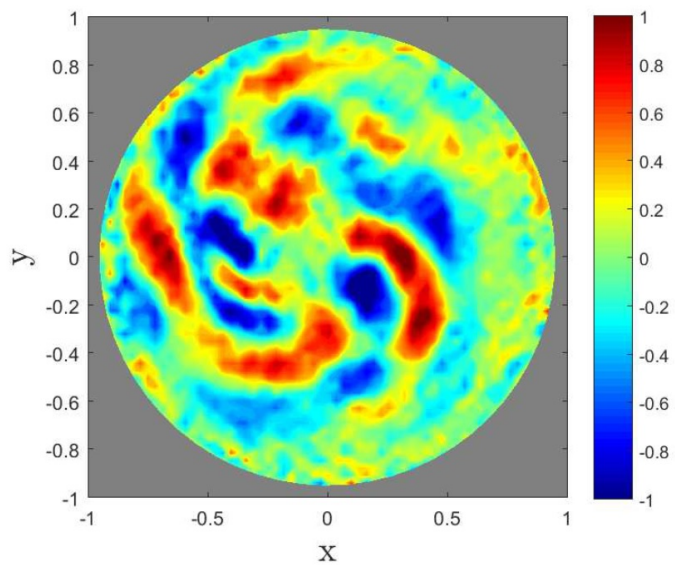

(a)

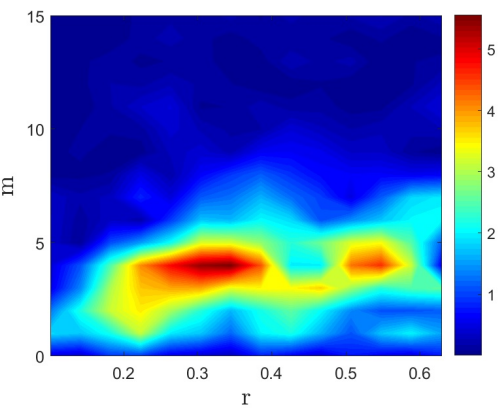

(c)

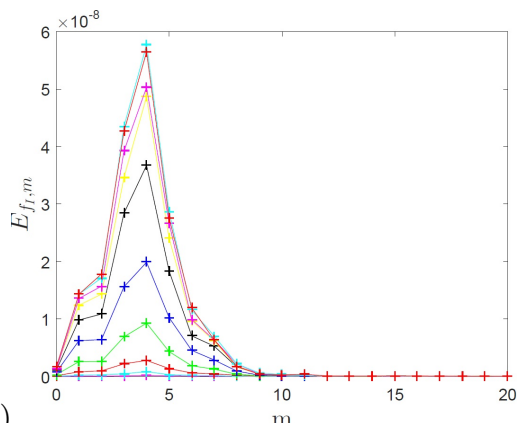

Figure 8. (a) Real part of the vorticity filtered at the frequency of the instability $2 \pi f_{I}=2.8$ as defined by Eq. (4.2). The Fourier transform is calculated over 16 rotation periods around $t / 2 \pi=100$, corresponding to the end of the exponentially growing stage. (b) Azimuthal spectrum of the vorticity as a function of the radius at $t / 2 \pi=100$. (c) Azimuthal spectrum integrated over $r$, as defined by (4.5) after 8 rotations (purple), after 24 rotations (cyan), after 40 rotations (red), after 56 rotations (green), after 72 rotations (blue), after 87 rotations (black), after 103 rotations (yellow), after 119 rotations (purple), after 135 rotations (cyan) and after 151 rotations (red). Here, $P o=-0.157, E=1.2 \times 10^{-4}$ and $h=0.065$.

where $\theta_{f}=0.72$ and $E=1.2 \times 10^{-4}$ leads to an empirical formula for the growth rate :

$$
\sigma=2.5\left(E^{1 / 5} \theta_{f}-7 \sqrt{E}\right) .
$$

with a $30 \%$ error on the numerical value 2.5 . Here, the viscous damping has been added in order to satisfy $\sigma=0$ at threshold. This viscous damping (equal to $-17.5 \sqrt{E}$ ) seems to be a bit large. Indeed, it is expected to be smaller than in the previous section (where it was equal to 13) since the azimuthal wavenumbers are lower. However, the Ekman number is larger here, such that the volumic viscous damping may not be negligible. The term $-17.5 \sqrt{E}$ could thus be rather equal to $10 \sqrt{E}+680 E$ for example, using ad hoc coefficients (see e.g. a detailed discussion in the closely related case of libration in Lemasquerier et al. (2017)). It is also possible that the boundary layer is turbulent in this regime, leading to a larger Ekman pumping and thus a larger damping rate of the inertial modes. This empirical formula is in good agreement with the prediction for the CSI, given by (4.11), where the second coefficient is equal to 9.5 rather than 7 .

To compare with the theory of the shear instability, the variation of the shear is calculated at $\theta_{f}=0.72$ using (4.13), leading to $\partial \epsilon / \partial \theta_{f}=-0.07 \pm \mathbf{2 0} \%$. It gives an 
empirical formula for the growth rate if it is due to the shear instability:

$$
\sigma=5.8 \epsilon-90 \sqrt{E} .
$$

with a $30 \%$ error on the numerical value 5.8. It means that the shear instability is probably not unstable for these parameters. Indeed, the theory predicts that the inviscid growth rate is equal to $0.6 \epsilon$ rather than $5.8 \epsilon$ (Kerswell 1993), and the viscous damping coefficient seems unreasonably large.

Finally, the variation of the ellipticity is calculated at $\theta_{f}=0.72$ using (4.14), leading to $\partial \beta / \partial \theta_{f}=-0.15 \pm \mathbf{2 0} \%$. It gives an empirical formula for the growth rate if it is due to the elliptic instability:

$$
\sigma=2.7 \beta-25 \sqrt{E} .
$$

with a $30 \%$ error on the numerical value 2.7 . It means that the elliptic instability is probably not unstable for these parameters. Indeed, the theory predicts an upper bound for the inviscid growth rate equal to $\beta$ rather than $2.7 \beta$ (Le Bars et al. 2010).

To conclude, the instability on this branch is probably due to the CSI. The elliptic and the shear instabilities seem to be stable for the second Busse's solution at this tilt angle.

\section{Conclusions}

In conclusion, our study confirms the presence of hysteresis between two uniform vorticity solutions around the resonance with the tilt-over mode, in good agreement with the analytical, viscous solution of Busse (1968) and the theoretical description of Cébron (2015). To the best of our knowledge, this is the first experimental validation of hysteresis for a moderate precession angle: our study thus expands the historical work of Malkus (1968) and the more recent one by Horimoto et al. (2018), considering precession close to $90^{\circ}$. Note that hysteresis has also been observed at this $90^{\circ}$ angle in a cylindrical geometry with aspect ratio 1 by Herault et al. (2015). In the first solution S1, the fluid rotation axis is only slightly inclined compared to the container rotation axis and the flow remains laminar in most of the explored range of parameters: its amplitude is then quantitatively similar to the Busse (1968) solution. For the second solution S2, the fluid rotation axis is more inclined and small-scale perturbations are almost systematically observed in addition to the global, large-scale, solid body rotation: a turbulent viscosity has then to be introduced for quantitative agreement with Busse (1968) solution. This turbulent viscosity is satisfyingly predicted by accounting for turbulent dissipation in the Ekman boundary layer, as described by Sous et al. (2013).

For each solution, we observe at low enough Ekman number an instability with a well defined spectral signature in space and time, suggesting a parametric resonance mechanism involving a forcing and two inertial waves of the rotating fluid. Using PIV measurements around the instability threshold, we have tried to decipher between the three possible origins for the forcing described in the litterature: the elliptical distortion of the circular streamlines of the inclined Busse's solution in the spheroid, as introduced by Kerswell (1993); the associated shearing of their centers, as also described by Kerswell (1993); and the conical shear strain generated from critical latitudes by Ekman layer eruption, as recently depicted in the spherical geometry by Lin et al. (2015) and in the spheroidal geometry by Horimoto et al. (2020). While the latter mechanism seems appropriate for both Busse's solutions, we are not in the position to completely discard the first two suggestions, especially for the first solution S1. In the range of experimentally accessible parameters, all mechanisms have indeed close growth rates and might superimpose: only a systematic and large-range exploration in terms of Ekman number 
will allow to reach a clear conclusion, since only the inviscid growth rate of the conical shear instability explicitly depends on the Ekman number. This is however experimentally challenging.

To finish with, trends for future experimental studies include the exploration of other ranges of Poincar number, to look for possible parametric instabilities away from the resonance with the tilt-over mode. It will also be very interesting to describe better the non-linear saturation of the excited instabilities and the shape of the excited turbulence, i.e. geostrophic vs. wave turbulence, as recently done in closely related configurations using libration forcing (Le Reun et al. 2019) and direct forcing (Brunet et al. 2020). This fundamental question will indeed have a strong influence on e.g. energy dissipation and magnetic field generation, both questions of great interest for planetary applications.

\section{Declaration of Interests}

The authors report no conflict of interest.

\section{Acknowledgment}

This work was supported by the European Research Council under the European Union's Horizon 2020 research and innovation program through Grant No. 681835FLUDYCO-ERC-2015-CoG.

\section{REFERENCES}

Boisson, J., CÉBron, D, Moisy, F \& Cortet, P-P 2012 Earth rotation prevents exact solidbody rotation of fluids in the laboratory. EPL (Europhysics Letters) 98 (5), 59002.

Brunet, M., Gallet, B. \& Cortet, P.-P. 2020 Shortcut to geostrophy in wave-driven rotating turbulence: the quartetic instability. Physical Review Letters 124 (12), 124501.

Bullard, E. C. 1949 The magnetic field within the earth. Proceedings of the Royal Society of London. Series A. Mathematical and Physical Sciences 197 (1051), 433-453.

Busse, FH. 1968 Steady fluid flow in a precessing spheroidal shell. J. Fluid Mech. 33, 739-752.

CÉBron, D 2015 Bistable flows in precessing spheroids. Fluid Dynamics Research 47, 025504.

CÉbron, D., Laguerre, R., Noir, J. \& Schaeffer, N. 2019 Precessing spherical shells: flows, dissipation, dynamo and the lunar core. Geophysical Journal International 219 (Supplement_1), S34-S57.

CÉbron, D., Le Bars, M. \& Meunier, P. 2010 Tilt-over mode in a precessing triaxial ellipsoid. Phys. Fluids 22 (11), 116601.

Gans, R. F. 1970 On the precession of a resonant cylinder. J. Fluid Mech. 476, 865-872.

Goepfert, O. \& Tilgner, A. 2016 Dynamos in precessing cubes. New Journal of Physics 18 (10), 103019.

Goto, S., Fujiwara, M. \& Yamato, M. 2011 Turbulence sustained in a precessing sphere and spheroids. In Seventh International Symposium on Turbulence and Shear Flow Phenomena. Begel House Inc.

Goto, S., Matsunaga, A., Fujiwara, M., Nishioka, M., Kida, S., Yamato, M. \& Tsuda, S. 2014 Turbulence driven by precession in spherical and slightly elongated spheroidal cavities. Physics of Fluids 26.

Grannan, AM, Le Bars, M, CÉBron, D \& Aurnou, JM 2014 Experimental study of globalscale turbulence in a librating ellipsoid. Physics of Fluids 26 (12), 126601.

Greenspan, H. P. 1968 The theory of rotating fluids. Cambridge University Press.

Herault, J., Gundrum, T., Giesecke, A. \& Stefani, F. 2015 Subcritical transition to turbulence of a precessing flow in a cylindrical vessel. Physics of Fluids 27 (12), 124102.

Horimoto, Yasufumi, Katayama, Atsushi \& Goto, Susumu 2020 Conical shear-driven parametric instability of steady flow in precessing spheroids. Phys. Rev. Fluids 5, 063901. 
Horimoto, Y., Simonet-Davin, G., Katayama, A. \& Goto, S. 2018 Impact of a small ellipticity on the sustainability condition of developed turbulence in a precessing spheroid. Phys. Rev. Fluids 3 (4), 044603.

Hough, SS 1895 The oscillations of a rotating ellipsoidal shell containing fluid. Philosophical Transactions of the Royal Society of London. A 186, $469 ? 506$.

KeRSWELL, RR 1996 Upper bounds on the energy dissipation in turbulent precession. Journal of Fluid Mechanics 321, 335-370.

Kerswell, R. R. 1993 The instability of precessing flow. Geophys. Astrophys. Fluid Dyn. 72, $107-144$.

KIDA, S. 2011 Steady flow in a rapidly rotating sphere with weak precession. J. Fluid Mech 680, 150-193.

KIDA, S. 2013 Instability by weak precession of the flow in a rotating sphere. Procedia IUTAM 7 pp. $180-192$.

KIDA, S. 2018 Steady flow in a rotating sphere with strong precession. Fluid Dynamics Research 50 (2), 021401.

KIDA, S. 2019 Instability by localized disturbances in critical region in a precessing sphere. Fluid Dynamics Research 52 (1), 015504.

KIDA, S. 2020 Steady flow in a rapidly rotating spheroid with weak precession: I. Fluid Dynamics Research 52 (1), 015513.

Lagrange, R., Meunier, P., Nadal, F. \& Eloy, C. 2011 Precessional instability of a fluid cylinder. J. Fluid Mech. 666, 104-145.

Le Bars, M., Cébron, D. \& Le Gal, P. 2015 Flows driven by libration, precession, and tides. Annu. Rev. Fluid Mech. 47, 163-193.

Le Bars, M., Lacaze, L., Le Dizès, S., Le Gal, P. \& Rieutord, M. 2010 Tidal instability in stellar and planetary binary systems. Physics of the Earth and Planetary Interiors 178 (1-2), 48-55.

Le Reun, T., Favier, B. \& Le Bars, M. 2019 Experimental study of the nonlinear saturation of the elliptical instability: inertial wave turbulence versus geostrophic turbulence. Journal of Fluid Mechanics 879, 296-326.

Lemasquerier, D, Grannan, AM, Vidal, J, Cébron, D, Favier, B, Le Bars, M \& Aurnou, JM 2017 Libration-driven flows in ellipsoidal shells. Journal of Geophysical Research: Planets 122 (9), 1926-1950.

Lin, Y., Marti, P. \& NoIR, J. 2015 Shear-driven parametric instability in a precessing sphere. Physics of Fluids $\mathbf{2 7}$.

Lorenzani, S. \& Tilgner, A. 2001 Fluid instabilities in precessing spheroidal cavities. J. Fluid Mech. 447, 111-128.

Lorenzani, S. \& Tilgner, A. 2003 Inertial instabilities of fluid flow in precessing spheroidal shells. JFM 492, 363-379.

Malkus, W. V. R. 1968 Precession of the Earth as the cause of geomagnetism. Science 160, 259-264.

Meunier, P., Eloy, C., Lagrange, R. \& Nadal, F. 2008 A rotating fluid cylinder subject to weak precession. J. Fluid Mech. 599, 405-440.

Meunier, P. \& Leweke, T. 2003 Analysis and minimization of errors due to high gradients in particule image velocimetry. Exp. Fluids.

Noir, J., Cardin, P., Jault, D. \& Masson, J. P. 2003 Experimental evidence of nonlinear resonance effects between retrograde precession and the tilt-over mode within a spheroid. Geophys. J. Int. 154, 407-416.

NoIR, J. \& CÉBRon, D. 2013 Precession-driven flows in non-axisymmetric ellipsoids. Journal of Fluid Mechanics 737, 412-439.

Noir, J., JAult, D. \& CARdin, P. 2001 Numerical study of the motions within a slowly precessing sphere at low Ekman number. J. Fluid Mech. 437, 283-29.

Poincaré, H. 1910 Sur la précession des corps déformables. Bull. Astro. 27, 257-264.

Sloudsky, T. 1895 De la rotation de la terre supposée fluide à son intérieur. Bull. Soc. Imp. Natur. Mosc. IX, 285-318.

Sous, D., Sommeria, J. \& Boyer, D. 2013 Friction law and turbulent properties in a laboratory ekman boundary layer. Physics of Fluids 25 (4), 046602. 
Stewartson, K. \& Roberts, P. H. 1963 On the motion of a liquid in a spheroidal cavity of a precessing rigid body. J. Fluid Mech. 17, 1-20.

Triana, SA, Zimmerman, DS \& LAThrop, DP 2012 Precessional states in a laboratory model of the earth's core. Journal of Geophysical Research: Solid Earth 117 (B4).

Vanyo, J. P. 1993 Rotating fluids in engineering and science. Dover, New York.

Vanyo, J. P., Wilde, P. \& Cardin, P. 1995 Experiments on precessing flows in the earth's liquid core. Geophys. J. Int. 121, 136-142.

Wu, Cheng-Chin \& Roberts, Paul H 2009 On a dynamo driven by topographic precession. Geophysical and Astrophysical Fluid Dynamics 103 (6), 467-501.

Wu, C. C. \& Roberts, P. H. 2011 High order instabilities of the poincaré solution for precessionally driven flow. Geophysical and Astrophysical Fluid Dynamics 105, 287-303.

Zhang, K., Chan, K. H. \& LiaO, X. 2014 On precessing flow in an oblate spheroid of arbitrary eccentricity. J. Fluid Mech. 743, 358-384. 